\title{
The subjective postural vertical in standing: Reliability and normative data for healthy subjects
}

\author{
Jeannine Bergmann • Monica-Antoanela Kreuzpointner • Carmen Krewer • \\ Stanislav Bardins • Andreas Schepermann • Eberhard Koenig • Friedemann Müller • \\ Klaus Jahn
}

Published online: 19 December 2014

(C) The Psychonomic Society, Inc. 2014

\begin{abstract}
Impaired verticality perception can cause falls, or even the inability to stand, due to lateropulsion or retropulsion. The internal estimate of verticality can be assessed through the subjective visual, haptic, or postural vertical (SPV). The SPV reflects impaired upright body orientation, but has primarily been assessed in sitting position. The internal representations of body orientation might be different between sitting and standing, mainly because of differences in somatosensory input for the estimation of SPV. To test the SPV during standing, we set up a paradigm using a device that allows movement in three dimensions (the Spacecurl). This study focused on the test-retest and interrater reliabilities of SPV measurements $(n=25)$ and provides normative values for the age range $20-79$ years $(n=60 ; 10$ healthy subjects per decade). The test-retest and interrater reliabilities for SPV measurements in standing subjects were good. The normality values ranged from $-1.7^{\circ}$ to $2.3^{\circ}$ in the sagittal plane, and from $-1.6^{\circ}$ to $1.2^{\circ}$ in the frontal plane. Minor alterations occurred with aging: SPV shifted backward with increasing age, and the variability of verticality estimates increased. Assessment of SPV in standing can be done with reliable results. SPV should next be used to test patients with an impaired sense of verticality, to determine its diagnostic value in comparison to established tools.
\end{abstract}

J. Bergmann $(\bowtie) \cdot$ C. Krewer $\cdot$ E. Koenig $\cdot$ F. Müller

Schoen Klinik Bad Aibling, Bad Aibling, Germany

e-mail: JBergmann@Schoen-Kliniken.de

J. Bergmann • M.-A. Kreuzpointner · C. Krewer · S. Bardins •

A. Schepermann $\cdot$ E. Koenig $\cdot$ F. Müller $\cdot$ K. Jahn

German Center for Vertigo and Balance Disorders (DSGZ),

Ludwig-Maximilians University, Munich, Germany

K. Jahn

Department of Neurology, Ludwig-Maximilians University of Munich, Munich, Germany
Keywords Spatial cognition · Motor control · Aging

The human sense of verticality is constructed and updated by integrating vestibular, somatosensory, and visual inputs (Barra et al., 2010). Verticality perception is impaired in different neurological disorders. Its disturbance-for example, in stroke - causes latero- or retropulsion and falls, both of which are major challenges for patient neurorehabilitation (Karnath \& Broetz, 2003; Manckoundia, Mourey, Pérennou, \& Pfitzenmeyer, 2008; Pérennou et al., 2008).

Different methods have been used to assess verticality perception: the subjective visual vertical (SVV; i.e., adjusting a bar that is visually compared with the gravitational vertical), the subjective haptic vertical (SHV; adjusting a bar to the gravitational vertical without visual control), and the subjective postural vertical (SPV; adjusting the body to the gravitational vertical). Most likely, SVV, SHV, and SPV test different but overlapping aspects of verticality control and yield complementary information (Pérennou et al., 2014). The SVV is the measure investigated most often. It is frequently used in the diagnosis of vestibular disorders, but is poorly correlated with postural impairment (Bonan et al., 2007; Karnath, Ferber, \& Dichgans, 2000; Pérennou et al., 2008). The SPV is altered in subjects with deficits of upright body orientation, both in the frontal and sagittal planes-for example, in subjects with pusher behavior or retropulsion after hemispheric lesions (Karnath et al., 2000; Manckoundia, Mourey, Pfitzenmeyer, Van Hoecke, \& Pérennou, 2007; Pérennou et al., 2008). Furthermore, the SPV is influenced by the aging process: With increasing age, the SPV shifts backward and body alignments are less accurate (Barbieri, Gissot, \& Pérennou, 2010). These age-related changes might be the consequences of a decline in sensory function (Manckoundia et al., 2008).

So far, SPV measurements have been mainly made with subjects in a sitting position, by using various motor-driven 
machines (e.g., Bisdorff, Wolsley, Anastasopoulos, Bronstein, \& Gresty, 1996; Karnath et al., 2000) or a nonmotorized paradigm, the so-called wheel paradigm (Pérennou, 2006). We hypothesized that the internal representations of body orientation might be different between sitting and standing, mainly because of differences in somatosensory inputs. Somatosensory inputs - that is, contact, proprioceptive, and visceral cues - play a major role in verticality perception (Bronstein, 1999). In sitting, several contact cues from the chair are available - for example, pressure cues on the back, under the buttocks, and on the back sides of the legs. These cues are not present during standing. Instead, upright stance involves pressure cues from the soles under the feet and somatosensory feedback from the ankle joints. Although sensory input from the lower extremities seems relatively unimportant for SPV estimation in sitting (Mazibrada et al., 2008), both contact and proprioceptive input might significantly contribute to the SPV in standing.

SPV assessment during standing might be especially relevant for postural disorders primarily affecting the standing posture. Several authors have reported that pusher behavior in its severe form is expressed in both sitting and standing positions. In a less severe form, or when the patient has progressed during rehabilitation, pusher behavior is no longer present in sitting, but continues in standing position (Babyar, Peterson, Bohannon, Pérennou, \& Reding, 2009; Bergmann et al., 2014; Premoselli, Cesana, \& Cerri, 2001). Considering that patients with pusher behavior attempt to align their body with an erroneous SPV (Pérennou et al., 2008), this suggests that the internal reference of verticality is represented differently during sitting and standing. Thus, for patients with deficient body orientation in standing, SPV assessment in sitting might not be able to detect the deficit. That is why we set up a paradigm to measure the SPV during standing using the Spacecurl. The Spacecurl is a cardanic suspension apparatus that so far has been used as a therapeutic approach for patients with neuropathy (Lauenroth, Knipping, \& Schwesig, 2012) or back pain (Müller, Schwesig, Leuchte, \& Riede, 2001). The purpose of this study was to investigate the reliability and normative values of SPV during standing using this paradigm. Healthy subjects were examined, and values in the sagittal and frontal planes were collected. A secondary objective was to investigate age-related differences in SPV during standing.

\section{Method}

\section{Subjects}

The reliability of SPV measurements was determined in 25 healthy subjects (age $34.4 \pm 9.7$ years [mean \pm standard deviation], 19 to 56 years [range]; 15 females, 10 males). In addition, the normative values were collected from 60 healthy subjects aged 20 to 79 years (ten subjects per decade). Exclusion criteria were acute cardiac disease, arterial aneurism, thrombosis, unstable spinal column, neuroses/psychoses, advanced pregnancy; body height $<145 \mathrm{~cm}$ and $>195 \mathrm{~cm}$, and body weight $>150 \mathrm{~kg}$. Subjects had to be free of any vestibular or balance deficit. Subjects $>50$ years of age underwent a neurological examination, including test of pallesthesia and a head-impulse test for vestibular function. The study was approved by the Ethics Committee of Ludwig-Maximilians University (LMU) Munich in accordance with the Declaration of Helsinki. All participants gave their written informed consent.

Apparatus and experimental procedure

The Spacecurl (Physio Boerse, Wittlich, Germany) is a cardanic suspension apparatus consisting of three concentric rings that allows rotation in three-dimensional space. The rings can be fixed so as to permit the rotation of the subject around each axis separately. The subject stands in the center of the apparatus (with the subject's hip approximately at the center of rotation) on a platform attached to the innermost ring, and is secured by padded holders on the hip (Fig. 1).

The settings of the platform and the holders were adjusted for each subject before making the first measurement and were retained for the following measurements. The level of the platform was chosen according to the body height of the subject. For a body height of $160 \mathrm{~cm}$, the platform was adjusted to $14 \mathrm{~cm}$. The platform level was lowered for taller persons and raised for smaller persons $(1 \mathrm{~cm}$ for $2 \mathrm{~cm}$ of body

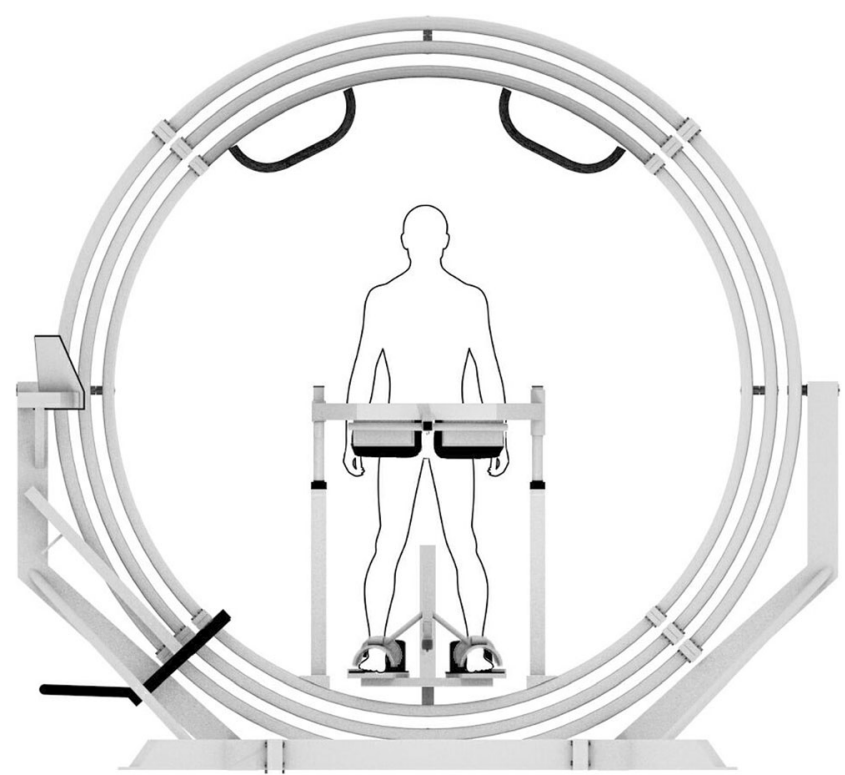

Fig. 1 Schematic illustration of a subject standing in the Spacecurl. The subject stands on a platform and is secured by padded holders at the hips and feet. The Spacecurl model is published with the kind permission of Klaus-Hendrik Wolf of the Peter L. Reichertz Institute for Medical Informatics, University of Braunschweig-Institute of Technology, and Hannover Medical School, Germany 
height). The padded holders were adjusted to the level of the iliac crests and of the lumbar lordosis of the back. These holders were tightly fixed in such a way that the subject stood upright. The feet were secured by padded brackets. These brackets were only loosely attached without affecting the load under the subject's feet.

An SPV measurement was made by an examiner and an assistant. The examiner gave standardized instructions and moved the rings of the Spacecurl. The assistant handled the computer. Before starting the measurement, the subject was instructed to stand in an upright body position, while placing his/her hands on the support frame right in front of the trunk. To rule out any visual input, the subject wore a pair of opaque goggles.

The SPV was first assessed in the sagittal plane and afterward in the frontal plane, using the method of magnitude production. Six trials per plane were conducted, with the start positions in random order $\left(12^{\circ}, 15^{\circ}\right.$, and $\left.18^{\circ}\right)$. From the start position, the Spacecurl was rotated back in the direction of the earth vertical or across until the subject had verbally identified the position that he or she felt to be upright. The subject was allowed to make small adjustments until he or she was satisfied that a vertical position had been reached. Subsequently the subject was tilted to the next start position. The Spacecurl was rotated manually as steadily and smoothly as possible by the examiner at a velocity of $1.0^{\circ}-1.5^{\circ}$ per second (feedback was provided on the computer screen).

Deviations from the earth vertical were measured with the Wireless Inertial Measurement Unit (IMU BT02-0300F05, Memsense, Rapid City, USA) placed on the support frame of the Spacecurl right in front of the subject, approximately on the level of the subject's body center. Data were transmitted wirelessly between the sensor and computer via the Bluetooth protocol and were recorded using a EyeSeeCam software module. The data were analyzed using a MATLAB-based program.

\section{Experimental designs}

To determine the test-retest and interrater reliability of the SPV measurements, the SPV was measured two times a day on two consecutive days. The study design is shown in Fig. 2. Measurement 1, Measurement 2, and Measurement 3 were made by the same examiner, whereas Measurement 4 was done by another examiner. The data from Measurements 1 and 2 were used to estimate test-retest reliability, and the data from Measurements 3 and 4 to estimate interrater reliability. Between both Measurements 1 and 2 and Measurements 3 and 4 , the subject had a standardized rest period of $20 \mathrm{~min}$ to relax on a chair. No feedback about his/her performance was given to the subject before the four SPV measurements were completed.

For the normative SPV values, only one session was necessary to measure the roll and pitch planes (as described above). The normative data were all assessed by the same two examiners.

Data and statistical analysis

The SPV was described in terms of the difference between the subject's perceived vertical and the gravitational vertical. In the sagittal plane, forward deviations of the SPV were given a positive sign, backward deviations a negative sign. In the frontal plane, rightward deviations were indicated by a positive sign and leftward deviations by a negative sign. The SPV error was obtained by averaging the six trials per measurement, and the SPV range was calculated as the difference between the maximum and minimum values of the six trials (Baccini, Paci, Del Colletto, Ravenni, \& Baldassi, 2014).

Test-retest reliability and interrater reliability were computed for SPV measurements in the sagittal and the frontal planes separately. To determine the consistency between measurements, the intraclass correlation coefficient (ICC) with the $95 \%$ confidence interval $(95 \% \mathrm{CI})$ was used. The $\operatorname{ICC}(2,6)$ model was applied to test the test-retest reliability, and the $\operatorname{ICC}(3,6)$ model was used to estimate the interrater reliability (Shrout \& Fleiss, 1979). The standard error of measurement (SEM) was defined as the square root of the mean squared error. To calculate the minimal detectable change (MDC), the

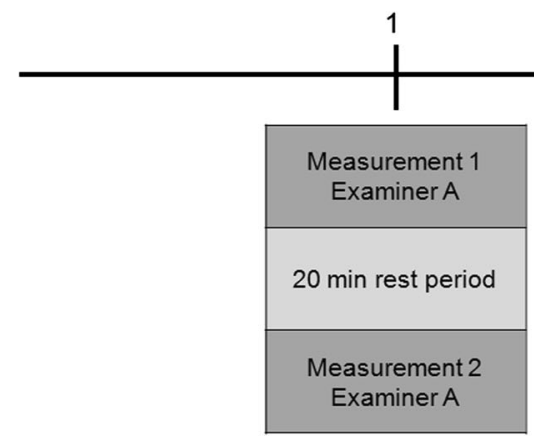

Fig. 2 Study design to determine the test-retest and interrater reliabilities of measurements of the subjective postural vertical using the Spacecurl. Four tests were performed on two consecutive days. Measurement 4 was

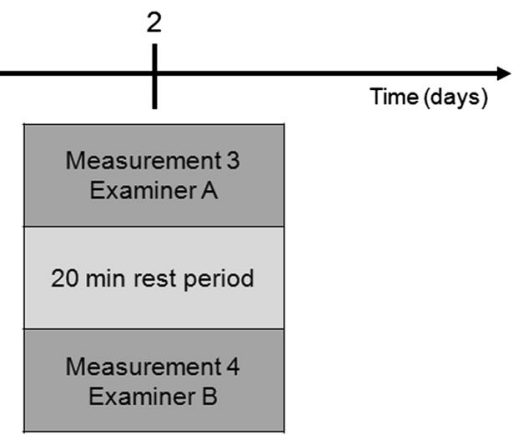

carried out by a different experimenter than Measurements 1, 2, and 3. To estimate test-retest reliability, Measurements 1 and 2 were compared; to estimate interrater reliability, Measurements 3 and 4 
SEM was multiplied by 1.96 and by the square root of 2 (Weir, 2005).

The degree of agreement between measurements was determined by calculating the mean difference between measures (d) and the $95 \%$ limits of agreement (LOA: $\mathrm{d} \pm 1.96 \mathrm{SD}$ ), displayed by Bland-Altman plots (Bland \& Altman, 1986). For comparison of the SPV errors and the SPV ranges between the age decades, univariate analyses of variance (ANOVAs) were performed for both planes, and post-hoc Tukey tests were applied. Correlation analyses between age and either the SPV error or the SPV range were carried out with the Pearson test. All calculations were considered significant at the $5 \%$ alpha level. Statistical analysis was performed using the statistical package SPSS Statistics 17.0.

\section{Results}

\section{Reliability}

All 25 subjects included in the reliability experiment completed the four SPV measurements. Table 1 presents ICCs with the $95 \%$ CIs, SEMs, and MDCs for estimation of the testretest and interrater reliabilities. Bland-Altman plots (Fig. 3) show mean differences between the measures and $95 \%$ LOAs for the test-retest and interrater reliabilities in the sagittal and frontal planes.

\section{Normative data and age dependency}

The mean SPV error and mean SPV range per age decade, and the results of the ANOVAs, are listed in Table 2. The largest difference between the age groups for the range of SPV in roll was found between subjects $40-49$ years and subjects 60-69 years of age, but this difference did not reach significance in the post-hoc test $(p=.064)$.

Table 1 Reliability parameters of subjective postural vertical measurements in the sagittal and frontal planes

\begin{tabular}{llll}
\hline & ICC $(95 \% \mathrm{Cl})$ & $\operatorname{SEM}\left(^{\circ}\right)$ & $\mathrm{MDC}\left(^{\circ}\right)$ \\
\hline Test-Retest & & & \\
$\quad$ Sagittal plane & $.70(.31-.87)$ & 0.7 & 1.9 \\
$\quad$ Frontal plane & $.73(.40-.88)$ & 0.5 & 1.3 \\
Interrater & & & \\
$\quad$ Sagittal plane & $.66(.23-.85)$ & 0.8 & 2.3 \\
$\quad$ Frontal plane & $.73(.39-.88)$ & 0.5 & 1.3 \\
\hline
\end{tabular}

ICC, intraclass correlation coefficient; 95\% CI, 95\% confidence interval; SEM, standard error of measurement; MDC, minimal detectable change
Because the SPV errors did not differ between the various age decades, the ranges of normality were calculated for the whole group of subjects from 20 to 79 years of age. The average SPV (mean $\pm S D$ ) for all subjects was $0.3^{\circ} \pm 1.0^{\circ}$ in the sagittal and $-0.2^{\circ} \pm 0.7^{\circ}$ in the frontal plane. Thus, the values of normality (mean $\pm 2 S D \mathrm{~s}$ ) in the sagittal plane ranged from $-1.7^{\circ}$ to $2.3^{\circ}$, and in the frontal plane, from $1.6^{\circ}$ to $1.2^{\circ}$.

Moderate, statistically significant correlations between age and SPV error and between age and SPV range were found for the sagittal plane (error: $r=-.262, p=.043$; range: $r=.385, p=.002)$, but not for the frontal plane ( $p>$ $.110)$. Scatterplots for the correlations in the sagittal plane are shown in Fig. 4.

\section{Discussion}

This was the first time that SPV measurements made with standing subjects have been evaluated for their test-retest and interrater reliabilities and that respective normative values have been given for the sagittal and frontal planes. We found overall good reliability in healthy subjects and minor changes with aging - that is, increased variance of the estimations. In the past, various devices have been used to measure the SPV, but mainly in sitting subjects. SPV assessment in a standing position might be relevant for postural impairments that primarily affect the standing posture.

\section{Reliability}

The present study revealed good reliability parameters for the SPV measurements with the Spacecurl in healthy subjects. The ICCs were .73 for both the test-retest and interrater reliabilities in the frontal plane, with a standard error of measurements of $0.5^{\circ}$. The reliability in the sagittal plane was slightly worse than in the frontal plane, in particular the interrater reliability. However, the reproducibility was still reasonable, with the standard error of measurements being smaller than $1^{\circ}$.

On the basis of our results, changes of the SPV are assumed to be clinically relevant if they are $\geq 1.3^{\circ}$ in the frontal plane and $\geq 1.9^{\circ}$ in the sagittal plane. These MDCs are similar to the LOAs illustrated in the Bland-Altman plots. Generally, the plots show good agreement, with very small differences between the measurements.

Nevertheless, the reliability assessed in healthy subjects is not necessarily applicable to those measured in very old people or patients with impaired balance. Assessments of test-retest and interrater reliability should therefore be performed in the respective sample of interest to confirm the potential clinical utility of these measures. 

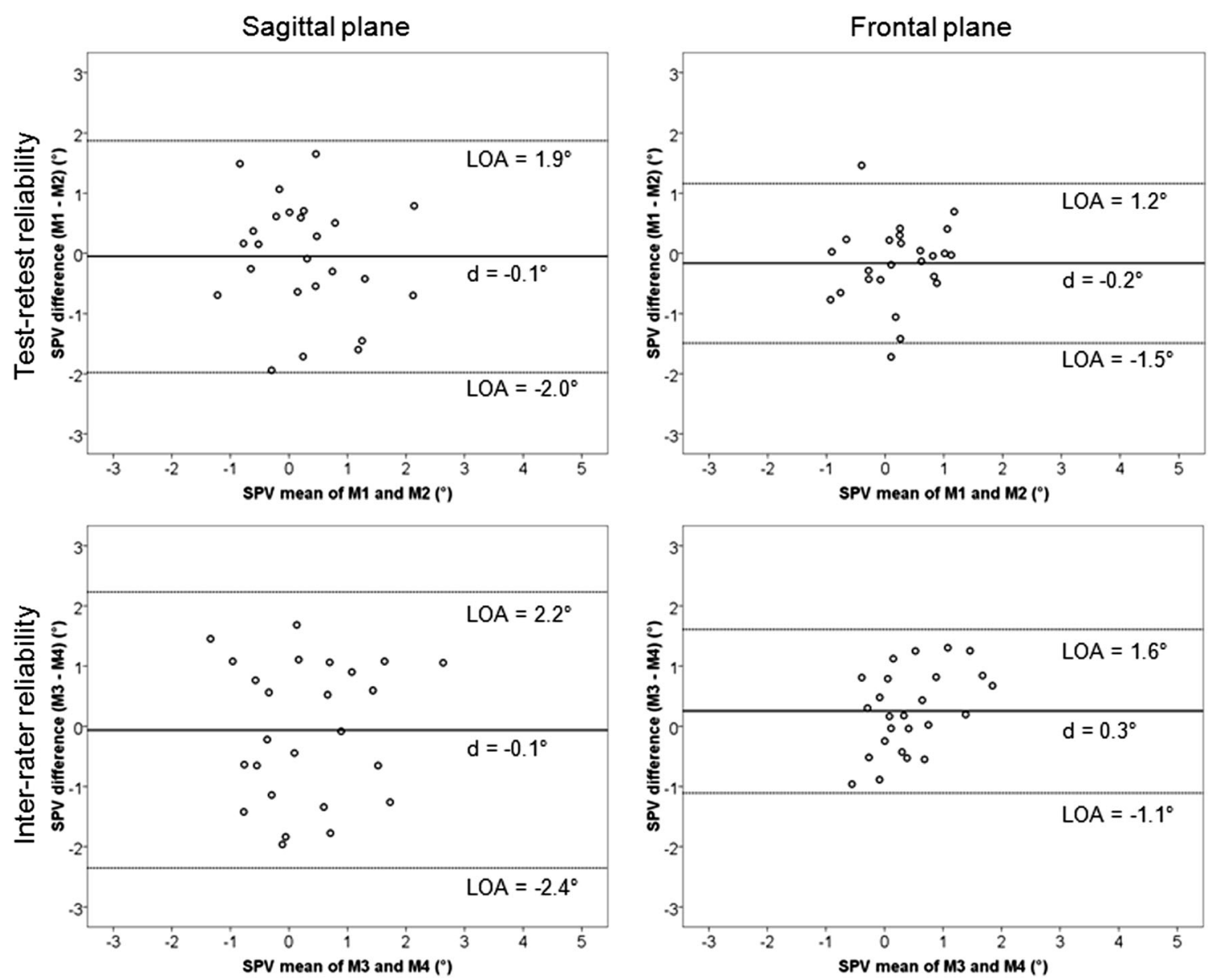

Fig. 3 Bland-Altman plots of the SPV in the sagittal and frontal planes: Differences between measurements versus the means of the measurements, with the mean difference (d) and 95\% limits of agreement (LOA). SPV, subjective postural vertical; M, measurement

Normative data

Normative values were collected over the age range. Since there was no significant difference in the SPV errors between decades, we calculated the ranges of normality for the whole

Table 2 Error and range (mean $\pm S D$ ) of subjective postural vertical for different age decades, with results of the ANOVAs

\begin{tabular}{llllll}
\hline & \multicolumn{2}{l}{ Sagittal Plane } & & \multicolumn{2}{l}{ Frontal Plane } \\
\cline { 2 - 3 } \cline { 5 - 6 } Age (years) & Error $\left(^{\circ}\right)$ & Range $\left(^{\circ}\right)$ & & Error $\left(^{\circ}\right)$ & Range $\left(^{\circ}\right)$ \\
\hline 20-29(7f) & $0.2 \pm 1.0$ & $3.2 \pm 1.3$ & & $-0.5 \pm 0.7$ & $2.2 \pm 1.0$ \\
$30-39(3 \mathrm{f})$ & $0.9 \pm 0.9$ & $4.3 \pm 1.9$ & & $-0.5 \pm 0.5$ & $2.6 \pm 1.2$ \\
$40-49(4 \mathrm{f})$ & $0.6 \pm 0.8$ & $4.4 \pm 1.5$ & & $-0.1 \pm 0.5$ & $3.6 \pm 1.6$ \\
$50-59(8 \mathrm{f})$ & $0.2 \pm 1.0$ & $5.0 \pm 1.4$ & & $0.0 \pm 0.6$ & $2.6 \pm 1.2$ \\
$60-69(5 \mathrm{f})$ & $0.1 \pm 0.6$ & $5.2 \pm 2.1$ & & $0.1 \pm 1.0$ & $2.1 \pm 0.8$ \\
$70-79(7 \mathrm{f})$ & $-0.2 \pm 1.2$ & $5.3 \pm 2.1$ & & $-0.3 \pm 0.7$ & $3.3 \pm 1.3$ \\
$F$ & 1.652 & 2.041 & & 1.871 & 2.532 \\
$p$ & 0.162 & 0.087 & & 0.115 & 0.039 \\
\hline
\end{tabular}

f, female group: $-1.7^{\circ}$ to $2.3^{\circ}$ in the sagittal plane, and $-1.6^{\circ}$ to $1.2^{\circ}$ in the frontal plane. For the sagittal plane, the range is similar to the values Barbieri et al. (2010) found in young adults in a sitting position $\left(<50\right.$ years; -2.4 to $\left.1.5^{\circ}\right)$. The only difference was that we found less backward tilt, most likely due to the different testing devices (see below). For older subjects $(\geq 50$ years), Barbieri et al. found a larger and more backwardshifted range of normality $\left(-4.0^{\circ}\right.$ to $\left.1.7^{\circ}\right)$. In the frontal plane, normative values for sitting SPV were given by Pérennou et al. (2008), who found a larger range than in our results. Similar to Pérennou et al. (2008), we found an almost symmetrical distribution of the normative values around the gravitational vertical in the frontal plane. This is in contrast to the sagittal plane, in which SPV values were distributed asymmetrically but with differences between standing and sitting. Whereas we observed an average slight forward tilt of the SPV in standing, Barbieri et al. found a backward tilt in sitting. As they discussed, it is likely that the backward-tilted SPV in sitting might be due to a methodological bias caused by the wheel paradigm that they used. When sitting in the wheel paradigm, the main contact points giving somatosensory information are under the buttocks and on the back. Since the 


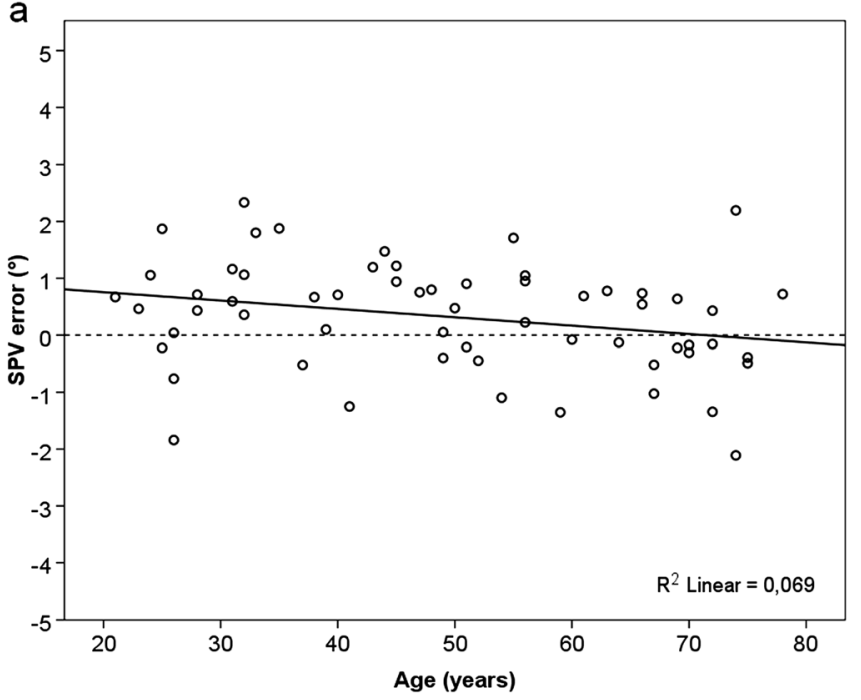

Fig. 4 Scatterplots illustrating the correlations between (a) subjective postural vertical (SPV) error and age and (b) SPV range and age, in the sagittal plane. The SPV error is slightly forward-tilted in younger subjects

internal estimate of verticality seems to be tilted to the side from which one gets more somatosensory information (Barra et al., 2010), the SPV might be shifted backward during sitting. In our paradigm, the main contact surfaces were almost symmetrical on the front and back, due to the padded holders on the hip as well as on the feet. There was thus no preponderance of one side.

\section{Age dependency}

Consistent with the findings of Barbieri et al. (2010), we found age-related changes of the SPV: a slight backward shift of the SPV error and a larger SPV range with increasing age. In the present study, the SPV error shifted from a small forward tilt in younger subjects toward the earth vertical with aging. In the work of Barbieri et al., the SPV shifted from an average slightly backward tilt in younger subjects to a more distinctly backward tilt in older subjects. The larger SPV range found in both studies in older subjects indicates increased uncertainty in verticality perception. Similarly, Bisdorff et al. (1996) observed larger sector widths of the SPV with aging - that is, a loss of sensitivity for the perception of body verticality. This reduced sensitivity may reflect an age-related decline of vestibular and somatosensory functions (Choy, Brauer, \& Nitz, 2003; Nusbaum, 1999). These sensory systems are involved in creating and updating the central representation of verticality (Barra et al., 2010). In particular, the somatosensory system is supposed to be important for the SPV-for example, to improve the stability of the verticality representation (Barbieri et al., 2010; Barra et al., 2010; Bringoux, Marin, Nougier, Barraud, \& Raphel, 2000).

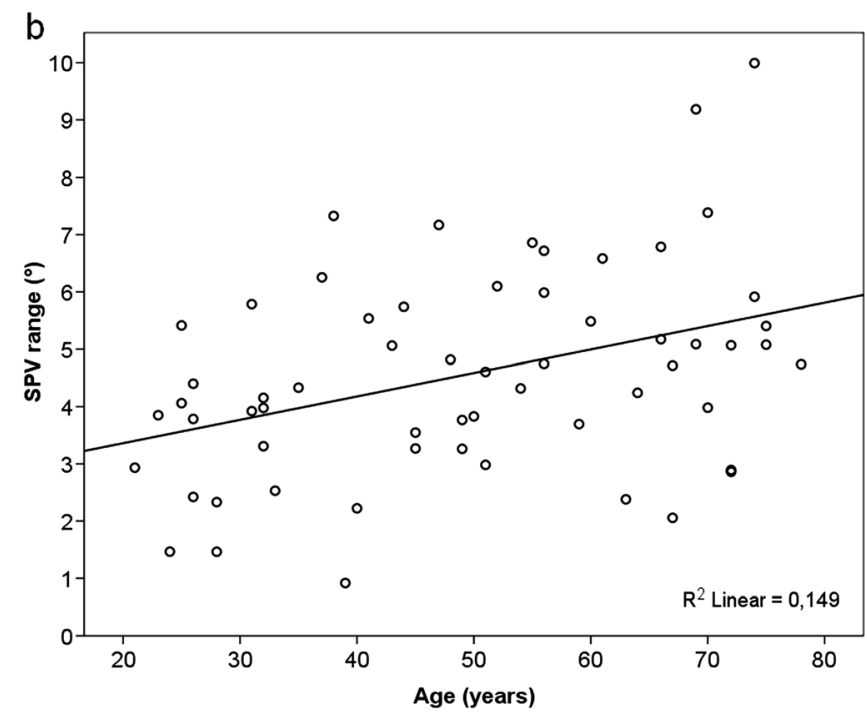

and approaches the earth vertical with increasing age. The SPV range increases with increasing age

Saeys et al. (2012) determined the influence of somatosensory loss on the perception of verticality in stroke patients during sitting. They differentiated between skin-related and joint-related somatosensory information and found a stronger relationship between the SPV and skin-related somatosensory input. In the Spacecurl, the contact area between the padded holders and the body is relatively small; however, the pattern of pressure at the hip and the pressure distribution under the feet might have affected the SPV measurements. Studies on patients with complete and partial somatosensory loss or on subjects with experimentally disturbed body sense (vibration) might help to determine the influences of somatosensory information on the SPV. When measuring SPV during sitting, Mazibrada et al. (2008) found a large error of the SPV in the frontal plane in a patient with Guillain-Barré syndrome and with severe symmetrical loss of peripheral sensation. The SPV accuracy considerably improved after recovery. In contrast, two patients with paralysis from Th 6-7 down did not show a significant error of the SPV. The authors concluded that somatosensory input from the trunk and the shoulders is especially important for the perception of verticality during sitting, whereas input from the lower limbs is less important, at least during sitting. For the SPV during standing, however, somatosensory input from the lower limbs and feet may play a major role.

A potential limitation of the Spacecurl as a measurement tool for the SPV is that the fixation on the hip not only provides the subject with somatosensory information but also forces the subject into an upright posture that might differ from the spontaneous subjective upright posture. Furthermore, the feet and hip fixations hamper postural control strategies such as the ankle or hip strategy in the sagittal 
plane. Nonetheless, the fixations are necessary to secure the subject. Since the Spacecurl was originally constructed for therapy, it provides no fixation of the trunk or head. For SPV measurements, its advantage is that no unnatural pressure cues are provided by holders or fixation straps on the upper body. However, the muscle activity needed to control the trunk and the head relative to gravity might increase the proprioceptive input available for SPV estimation, and might consequently lead to more precise and robust SPV measurements (as in the natural condition). This might account for the rather small variability in our data. Previous studies assessing the SPV had restrained the trunk and head and had allowed for rather less postural activity. In our study, the trunk and head were free to move, and postural control was needed to a greater extend. It remains to be determined whether complete restraint or more free standing is better suited to measure the inner representation of body orientation in space.

Another limitation, especially in comparisons with studies assessing the SPV in sitting, is the comparatively small tilt of the start positions. A maximum tilt angle of $18^{\circ}$ was chosen, because larger angles were hardly tolerable by several subjects, due to the upper body being free to move. Larger angles in these subjects caused fear and undesired postural reactions. Future measurements with patients should determine whether these start positions are suitable for detecting SPV deviations in patients with severely impaired verticality perception.

In conclusion, we found precise and reliable SPV estimations for healthy subjects in standing using the Spacecurl. We found on average a slightly forward-tilted SPV, which approached the earth vertical with increasing age. The clinimetric properties of the SPV in standing have to be further investigated in patients with deficits of postural control and/or upright body orientation. SPV measurements in patients with somatosensory, vestibular, or central disorders will lead to a better understanding of the pathways forming the inner model of verticality perception. The Spacecurl is a promising tool for these kinds of experiments.

Author note This work was supported by funds from the German Federal Ministry of Education and Research (Grant No. BMBF 01EO0901). We thank Judy Benson for copyediting the manuscript, Klaus-Hendrik Wolf for providing the graphical Spacecurl model (Fig. 1), and Andy Chung for editing the graphic.

\section{References}

Babyar, S. R., Peterson, M. G., Bohannon, R., Pérennou, D., \& Reding, M. (2009). Clinical examination tools for lateropulsion or pusher syndrome following stroke: A systematic review of the literature. Clinical Rehabilitation, 23, 639-650. doi:10.1177/ 0269215509104172
Baccini, M., Paci, M., Del Colletto, M., Ravenni, M., \& Baldassi, S. (2014). The assessment of subjective visual vertical: Comparison of two psychophysical paradigms and age-related performance. Attention, Perception, \& Psychophysics, 76, 112-122. doi:10. 3758/s13414-013-0551-9

Barbieri, G., Gissot, A. S., \& Pérennou, D. (2010). Ageing of the postural vertical. Age, 32, 51-60. doi:10.1007/s11357-009-9112-5

Barra, J., Marquer, A., Joassin, R., Reymond, C., Metge, L., Chauvineau, V., \& Pérennou, D. (2010). Humans use internal models to construct and update a sense of verticality. Brain, 133, 3552-3563. doi:10. 1093/brain/awq311

Bergmann, J., Krewer, C., Rieß, K., Müller, F., Koenig, E., \& Jahn, K. (2014). Inconsistent classification of pusher behaviour in stroke patients: A direct comparison of the Scale for Contraversive Pushing and the Burke Lateropulsion Scale. Clinical Rehabilitation, 28, 696-703. doi:10.1177/ 0269215513517726

Bisdorff, A. R., Wolsley, C. J., Anastasopoulos, D., Bronstein, A. M., \& Gresty, M. A. (1996). The perception of body verticality (subjective postural vertical) in peripheral and central vestibular disorders. Brain, 119, 1523-1534.

Bland, J. M., \& Altman, D. G. (1986). Statistical methods for assessing agreement between two methods of clinical measurement. Lancet, 1 , 307-310.

Bonan, I. V., Hubeaux, K., Gellez-Leman, M. C., Guichard, J. P., Vicaut, E., \& Yelnik, A. P. (2007). Influence of subjective visual vertical misperception on balance recovery after stroke. Journal of Neurology, Neurosurgery \& Psychiatry, 78, 49-55. doi:10.1136/ jnnp.2006.087791

Bringoux, L., Marin, L., Nougier, V., Barraud, P. A., \& Raphel, C. (2000). Effects of gymnastics expertise on the perception of body orientation in the pitch dimension. Journal of Vestibular Research, 10, 251-258.

Bronstein, A. M. (1999). The interaction of otolith and proprioceptive information in the perception of verticality: The effects of labyrinthine and CNS disease. Annals of the New York Academy of Sciences, 871, 324-333.

Choy, N. L., Brauer, S., \& Nitz, J. (2003). Changes in postural stability in women aged 20 to 80 years. Journals of Gerontology, 58A, 525530.

Karnath, H. O., \& Broetz, D. (2003). Understanding and treating "pusher syndrome.". Physical Therapy, 83, 1119-1125.

Karnath, H. O., Ferber, S., \& Dichgans, J. (2000). The origin of contraversive pushing: Evidence for a second graviceptive system in humans. Neurology, 55, 1298-1304.

Lauenroth, A., Knipping, S., \& Schwesig, R. (2012). Vestibular disorders. Effects of sensorimotor training on postural regulation and on recovery process. HNO, 60, 692-699. doi:10. 1007/s00106-011-2479-5

Manckoundia, P., Mourey, F., Pérennou, D., \& Pfitzenmeyer, P. (2008). Backward disequilibrium in elderly subjects. Clinical Interventions in Aging, 3, 667-672.

Manckoundia, P., Mourey, F., Pfitzenmeyer, P., Van Hoecke, J., \& Pérennou, D. (2007). Is backward disequilibrium in the elderly caused by an abnormal perception of verticality? A pilot study. Clinical Neurophysiology, 118, 786-793. doi:10.1016/j.clinph. 2006.11.274

Mazibrada, G., Tariq, S., Pérennou, D., Gresty, M., Greenwood, R., \& Bronstein, A. M. (2008). The peripheral nervous system and the perception of verticality. Gait \& Posture, 27, 202-208. doi:10.1016/ j.gaitpost.2007.03.006

Müller, K., Schwesig, R., Leuchte, S., \& Riede, D. (2001). Koordinationstraining und Lebensqualität-Eine Längsschnittuntersuchung bei Pflegepersonal mit Rückenschmerzen [Coordinative treatment and quality of life-A randomised trial of nurses with back pain]. Gesundheitswesen, 63, 609-618. doi:10.1055/s-2001-17872 
Nusbaum, N. J. (1999). Aging and sensory senescence. Southern Medical Journal, 92, 267-275.

Pérennou, D. (2006). Postural disorders and spatial neglect in stroke patients: A strong association. Restorative Neurology and Neuroscience, 24, 319-334.

Pérennou, D. A., Mazibrada, G., Chauvineau, V., Greenwood, R., Rothwell, J., Gresty, M. A., \& Bronstein, A. M. (2008). Lateropulsion, pushing and verticality perception in hemisphere stroke: A causal relationship? Brain, 131, 2401-2413. doi:10. 1093/brain/awn170

Pérennou, D., Piscicelli, C., Barbieri, G., Jaeger, M., Marquer, A., \& Barra, J. (2014). Measuring verticality perception after stroke: Why and how? Clinical Neurophysiology, 44, 25-32. doi:10.1016/j. neucli.2013.10.131
Premoselli, S., Cesana, L., \& Cerri, C. (2001). Pusher syndrome in stroke: Clinical, neuropsychological and neurophysiological investigation. European Journal of Physical and Rehabilitative Medicine, 37, $143-151$.

Saeys, W., Vereeck, L., Truijen, S., Lafosse, C., Wuyts, F. P., \& Van de Heyning, P. (2012). Influence of sensory loss on the perception of verticality in stroke patients. Disability and Rehabilitation, 34, 1965-1970. doi:10.3109/09638288.2012.671883

Shrout, P. E., \& Fleiss, J. L. (1979). Intraclass correlations: Uses in assessing rater reliability. Psychological Bulletin, 86, 420-428. doi:10.1037/0033-2909.86.2.420

Weir, J. P. (2005). Quantifying test-retest reliability using the intraclass correlation coefficient and the SEM. Journal of Strength \& Conditioning Research, 19, 231-240. 